\title{
Future Materials Research Trend
}

\author{
Kenji Uchino* \\ Office of Naval Research, Japan \& Int'l Center for Actuators \& Transducers, The Penn State University, University Park, USA
}

Received: February 08, 2014; Accepted: February 24, 2014; Published: February 25, 2014

*Corresponding author: ONR Global-Asia, Office of Naval Research, Roppongi, Minato-Ku, Tokyo 106-0032, Japan; \& Int'l Center for Actuators \& Transducers, The Penn State University, University Park, PA 16802, USA, Tel: 03-6385-3250; Fax: 03-6385-3254; E-mail: Kenji.Uchino.civ@mail.mil

\section{Editorial}

SOJ Materials Science \& Engineering (SOJMSE) aims to enhance the international exchange of scientific activities. It reports principally the achievements of materials science and engineering all over the world, putting the stress on the original research papers, review articles invited by editor, letters and research notes with novelty as well as brief of scientific achievement, covering a broad spectrum of materials science and technology. The scope of the journal includes the research advances involving in engineering of metallic materials, biomaterials, inorganic nonmetallic materials and composite materials.

The author would like to discuss the "MUST" strategies, which the materials research engineers need to keep in their minds in these decades.

After the World War II, Japanese government set the fourChinese-character slogan "heavier, thicker, longer, and larger" in 1960s; that is, manufacturing heavier ships, thicker steel plates, constructing longer buildings, and larger power plants (dams) were the key strategies for recovering from the ruins of World War II (i.e., Domestic Politics). This societal mood made the author choose Electrical Engineering in the university to become a water-dam engineer. Though the Japanese people became wealthy, subsidiary effects started to surface: i.e., "industrial pollution". The southern part of the Sea of Japan was contaminated by the heavy metal (mercury) disposal by a chemical fertilizer manufacturer, which created thousands of "Angel Babies (missing/dislocated arm disorder)" in that wide area. Steel industries produced air pollution and "asthma" even for small kids. Traffic congestion generated severe acoustic noise even in suburban areas. One of the most technological industries, nuclear power plants, leaked hazardous radio-active wastes multiple times. The "melt-down" accident of Three-Mile-Island, Pennsylvania happened in 1979. The target in materials research in this period was focused on construction materials such as steels, cements and plastics.

A completely opposite slogan started in the 1980s; that is, "lighter, thinner, shorter, and smaller". Printers and cameras became lighter in weight, thinner computers and TVs (flat panel) gained popularity, printing time and information transfer period became shorter, and air-conditioners and tape recorders ("Walk-man" by SONY) were smaller. Because of this societal mood, the author, as a young university professor then, started working on compact "piezoelectric actuators \& motors". Though the serious industrial pollution diminished gradually during this period in proportion to the country power (high GDP per person), different subsidiary effects started: (1) "greenhouse effect" and "global warming" due to $\mathrm{CO}_{2}$ gas generated by over-produced automobiles, (2) energy crisis due to overconsumption of energy and lack of fossil energy sources (oil), in addition to the political mismanagement, and (3) population growth due to advanced medical technologies in developing countries and less children \& more seniors in matured countries. Longer life time is welcomed by individuals (now the average age for Japanese female is approaching to 87, male, 82 already; the world-eldest). However, over-population of humans will create an imbalance against other animals/natures, and senior population, in particular, causes societal and economic problems (pension, health insurance, work force etc.). This economically progressing period emphasized the materials research in terms of mass-production, lower manufacturing cost with reasonable performance. Semiconductor seemed to be in the main stream in research. $60 \%$ of the publications were on the semiconductors in most of the materials-related academic journals.

When the 21st century began, as a consequent result, environmental degradation, resource depletion, and food famine have become major problems. Global regulations are strongly called, and the government-initiated technology, that is, "politicoengineering" has become further important again in order to overcome the regulations. The author is proposing a new fourChinese-character keyword for the era of "politico-engineering", "cooperation, protection, reduction, and continuation". Global coordination and international cooperation in standardization of internet systems and computer cables became essential to accelerate the mutual communication. The Kyoto Protocol in December 1997 is an international agreement linked to the United Nations Framework Convention on Climate Change. Its major feature is to set binding targets for 37 industrialized countries and the European community for reducing greenhouse gas emission. Protection of the territory and environment from the enemy or natural disaster, and of infectious disease spread is mandatory. Reduction of toxic materials such as lead, 
heavy metals, dioxin, and of the use of resources and energy consumption is also the key, and the society continuation, i.e., status quo or Sustainable Society, is important to promote. Thus, the current materials research is seeking alternative materials (environmentally friendly materials) for replacing toxic ones, bio/medical materials and materials related with energy generation, in particular, nuclear power plant protection. The author's research focuses are now piezoelectric/pyroelectric energy harvesting, Pb-free piezoelectric materials and defenserelated actuators/sensors.

As a concluding remark, the author's recommendation is to learn global and domestic political strategies for 10 years ahead, and to reflect the materials development according to this direction. 